\title{
Inertial Manifolds for Generalized Higher-Order Kirchhoff Type Equations
}

\author{
Guoguang $\operatorname{Lin}^{1}$, Shuangyan $\mathrm{Li}^{1}$ \\ ${ }^{1}$ School of Mathematics and Statistics, Yunnan University, Kunming, Yunnan \\ Correspondence: Guoguang Lin, School of Mathematics and Statistics, Yunnan University, Kunming, Yunnan
}

Received: August 13, 2020 Accepted: September 7, 2020 Online Published: September 21, 2020

doi:10.5539/jmr.v12n5p67 URL: https://doi.org/10.5539/jmr.v12n5p67

\begin{abstract}
The existence of inertial manifolds for higher-order Kirchhoff type equations with strong damping terms is studied. The Hadamard graph norm conversion method is used for obtaining the existence of inertial manifolds for this kind of equations under certain spectral intervals.
\end{abstract}

Keywords: inertial manifold, spectral interval condition, figure van, higher-order Kirchhoff equation

\section{Introduction}

In the study of the long-term dynamic behavior of infinite dimensional dynamical systems, the inertial manifold occupies an important position. It is a finite dimensional invariant Lipschitz manifold and attracts all solution orbitals with exponential rate in the phase space of the system [1-3]. It plays an important role in both finite dimensional dynamical systems and infinite dimensional dynamical systems.Because it occupies an important position, many scholars have studied the existence and attraction of inertial manifolds, the finite-dimensional properties, and the related problems of approximate inertial manifolds and delay. Guoguang Lin and Jingzhu Wu [4] studied the existence of inertial manifolds of the low order Bousinesq equation with strongly damped term at that time, and the equation is

$$
\left\{\begin{array}{l}
u_{t t}-\alpha \Delta u_{t}-\Delta u+u^{2 k+1}=f(x, y),(x, y) \in \Omega, \\
u(x, y, 0)=u_{0}(x, y),(x, y) \in \Omega, \\
u(x, y, t)=u(x+\pi, y, t)=u(x, y+\pi, t)=0,(x, y) \in \Omega .
\end{array}\right.
$$

Zhicheng Zhang and Guoguang Lin [5] study the following fourth order strongly damped time-delay wave equations:

$$
\left\{\begin{array}{l}
u_{t t}-\varepsilon \Delta u_{t}-\Delta u+\Delta^{2} u=f\left(u_{t}\right), t>0 \\
u_{0}(\Theta)=u^{0}(\Theta), \Theta \in[-r, 0],\left.\partial_{t}\right|_{t=0}=u .
\end{array}\right.
$$

The inertial manifolds of the above equations under the assumption of delay term from distribution are studied. In this paper, based on this, will rise again, order space optimization, joined the high order structural damping, under certain assumptions prove Kirchhoff type generalized high order equation of inertial manifolds exist. More on the Kirchhoff equation of inertial manifolds, see reference[7-12].

This paper study the initial boundary value problems of the following Kirchhoff type equations:

$$
\left\{\begin{array}{l}
u_{t t}+\left(1+\int_{\Omega}\left|D^{m} u\right|^{p} d x\right)^{r}(-\Delta)^{m} u+\Delta^{2 m} u+\beta(-\Delta)^{m} u_{t}=f(x), \\
u(x, t)=0, \frac{\partial^{j} u}{\partial v^{i}}=0, i=1,2, \cdots, 2 m-1, x \in \partial \Omega, t>0, \\
u(x, 0)=u_{0}(x), u_{t}(x, 0)=u_{1}(x), x \in \Omega \subset R^{n}
\end{array}\right.
$$

Where $r \geq 0, m \geq 1, \beta>0 . \Omega$ is the bounded region with smooth boundary $\partial \Omega$ in $R^{n} . f(x)$ is the external force term. $v^{i}$ is the external normal vector, $\Delta^{2 m} u$ is the structural damping term, $\beta(-\Delta)^{m} u_{t}$ is the structure damping term. $(1+$ $\left.\int_{\Omega}\left|D^{m} u\right|^{p} d x\right)^{r}(-\Delta)^{m} u$ is the rigid term. And the assumptions about the rigid term will be given late.

\section{Prepare}

For the convenience, this paper defines Spaces and symbols as follows :

$f=f(x), D=\nabla, H=L^{2}(\Omega), H_{0}^{2 m}=H^{2 m}(\Omega), V_{1}=H_{0}^{2 m}(\Omega) \times H(\Omega), V_{2}=H_{0}^{2 m+k} \times H_{0}^{k}(\Omega)(k=0,1,2, \cdots, 2 m), C_{0}$ is constant. Respectively, $(\cdot, \cdot)$ and $\|\cdot\|$ represent the inner product and norm of $\mathrm{H}$. That is $(u, v)=\int_{\Omega} u(x) v(x) d x,(u, v)=$ 
$\|u\|^{2}$.And then let's define the norm and the inner product of the spaces $V_{1}$ and $V_{2}: \forall U_{i} \in\left(u_{i}, v_{i}\right) \in V_{i}, i=1,2$, we have

$$
\begin{aligned}
& \left(U_{1}, U_{2}\right)=\left(D^{m} u_{1}, D^{m} u_{2}\right)+\left(v_{1}, v_{2}\right), \\
& \|U\|_{V_{1}}^{2}=(U, U)_{V_{1}}=\left\|D^{m} u\right\|^{2}+\|v\|^{2}, \\
& \|U\|_{V_{1}}^{2}=(U, U)_{V_{2}}=\left\|D^{m} u\right\|^{2}+\|v\|^{2} .
\end{aligned}
$$

$g\left(\left\|D^{m} u\right\|_{p}^{p}\right)=\left(1+\int_{\Omega}\left|D^{m} u\right|^{p} d x\right)^{r}$ meets the following conditions:

(a) $\left\|D^{m} u\right\|_{L^{p}} \leq C\|D u\|_{L^{2}}^{\alpha}\|u\|_{H^{2 m}}^{1-\alpha}$, where $\alpha=\left(\frac{1}{p}-\frac{n}{m}-\frac{1}{4 m}\right) /\left(\frac{1}{2}-\frac{1}{n}-\frac{1}{4 m}\right)$.

(b)Writing $g\left(\left\|D^{m} u\right\|_{p}^{p}\right)=\left(1+\int_{\Omega}\left|D^{m} u\right|^{p} d x\right)^{r}$ by (a) we get

$\kappa_{0} \leq g\left(\left\|D^{m} u\right\|_{p}^{p}\right) \leq \kappa_{1}, \kappa=\left\{\begin{array}{l}\kappa_{0}, \frac{d}{d t}\left\|D^{m+k} u\right\|_{p}^{p} \geq 0, \\ \kappa_{1}, \frac{d}{d t}\left\|D^{m+k} u\right\|_{p}^{p}<0 .\end{array}\right.$ And $\kappa_{0} \geq 1, \kappa_{1}, C,\|u\|_{H^{2 m}}$ is constant.

Definition 2.1[2] One says that inertial manifolds $\mu$ are finite-dimensional manifolds that need to satisfy the following these properties:

(i) $\mu$ is a lipschitz manifold and finite dimension;

(ii) $\mu$ is a positive invariant set, that is $S(t) \mu \subset \mu, \forall t>0$;

(iii) The $\mu$ exponential attracts all the solution orbitals.

Definition 2.2[2] Assume $A_{1}: X \rightarrow X$ is an operator and $F \in C_{b}(X, X)$ satisfy the following inequalities

$$
\|F(U)-F(V)\|_{X} \leq l_{F}\|U-V\|_{X}(U, V \in X)
$$

Suppose the point spectrum of operator $A_{1}$ can be divided into two parts $\sigma_{1}$ and $\sigma_{2}$, and which $\sigma_{1}$ is finite,

$$
\Lambda_{1}=\sup \left\{\operatorname{Re} \lambda \mid \lambda \in \sigma_{1}\right\}, \Lambda_{2}=\inf \left\{\operatorname{Re} \lambda \mid \lambda \in \sigma_{2}\right\} .
$$

And

$$
X_{i}=\operatorname{span}\left\{\omega_{j} \mid j \in \sigma_{i}\right\}, i=1,2 .
$$

So

$$
\Lambda_{2}-\Lambda_{1}>4 l_{F},
$$

We have orthogonal decomposition

$$
X=X_{1} \oplus X_{2} .
$$

and continuous mapping

$$
P_{1}: X \rightarrow X_{1}, P_{2}: X \rightarrow X_{2} .
$$

Lemma 2.1[6]Suppose that the eigenvalues $\mu_{j}^{ \pm}(j \geq 1)$ are nonsubtractive, and for all $m \in N$, when $N \geq m, \mu_{N}^{-}$and $\mu_{N+1}^{-}$ are consecutive adjacent values.

\section{Inertial Manifolds}

Equation (1) is equivalent to the following first-order evolution equation

$$
U_{t}+\widetilde{A} U=F(U)
$$

where $U=(u, v), v=u_{t}$,

$$
\begin{gathered}
\widetilde{A}=\left(\begin{array}{cc}
0 & -I \\
(-\Delta)^{2 m} & \beta(-\Delta)^{m}
\end{array}\right) . \\
F(U)=\left(\begin{array}{c}
0 \\
f(x)-\left(1+\left\|D^{m} u\right\|_{p}^{p}\right)^{r}(-\Delta)^{m} u
\end{array}\right) .
\end{gathered}
$$




$$
D(\widetilde{A})=\left\{u \in H^{2 m+k} \mid u \in L^{2},(-\Delta)^{m} u \in H^{2 m+k}\right\} \times H^{m} .
$$

The graph norm defined in $X$ by the dot product

$$
(U, V)_{X}=\left((-\Delta)^{m} u,(-\Delta)^{m} \bar{y}\right)+(v, \bar{z})
$$

where $U=(u, v), V=(y, z) \in X, u, y \in H^{2 m+k}(\Omega) ; v, z \in H^{2 m+k}(\widetilde{\Omega}) . \bar{y}, \bar{z}$ respectively represent conjugate of $y, z$. Obviously, the operator defined in equation (11) is monotone. For $U \in D(\widetilde{A})$, have

$$
(\widetilde{A} U, U)_{X}=-\left((-\Delta)^{m} v,(-\Delta)^{m} \bar{u}\right)+\left((-\Delta)^{2 m} u+\beta(-\Delta)^{m} v, \bar{v}\right)=\beta\left\|D^{m} v\right\|^{2} \geq 0 .
$$

So $(\widetilde{A} U, U)_{X}$ is a non-negative real number.

To determine the eigenvalues of $\widetilde{A}$,consider the following eigenvalue equation

$$
\widetilde{A}(U)=\lambda U, U=(u, v) \in X .
$$

That is

$$
\left\{\begin{array}{l}
-v=\lambda u \\
(-\Delta)^{2 m} u+\beta(-\Delta)^{m} v=\lambda v
\end{array}\right.
$$

By substituting the first equation of fomular (17) into the second equation of equation (17), we obtained

$$
\left\{\begin{array}{l}
\lambda^{2} u+(-\Delta)^{2 m} u-\beta \lambda(-\Delta)^{m} u=0 \\
\left.u\right|_{\partial \Omega}=\left.(-\Delta)^{m} u\right|_{\partial \Omega}=0
\end{array}\right.
$$

Used for the inner product of the first expression in equation (18), we get

$$
\lambda^{2}\|u\|^{2}+\left\|(-\Delta)^{m} u\right\|^{2}-\beta \lambda\left\|(-\Delta)^{m} u\right\|^{2}=0 .
$$

Equation (19) is regarded as a quadratic equation with one unknown about $\lambda$, so there is

$$
\lambda_{k}^{ \pm}=\frac{\beta \delta_{k} \pm \sqrt{\beta^{2} \delta_{k}^{2}-4 \delta_{k}^{2}}}{2} .
$$

Where $\delta_{k}$ is the eigenvalue of $(-\Delta)^{m}$ in $H^{2 m}(\Omega)$. If $\beta^{2} \geq 4$, then all the eigenvalues of $\widetilde{A}$ are positive real numbers, and the corresponding eigenvector has the form of $U_{k}^{ \pm}=\left(u_{k},-\lambda_{k}^{ \pm} u_{k}\right)$.As for formula (13), in order to facilitate the use of the following, the following marks will be made. For all $k \geq 1$, we get

$$
\left\|D^{m} u_{k}\right\|=\sqrt{\delta_{k}},\left\|u_{k}\right\|^{2}=1,\left\|(-\Delta)^{2 m} u_{k}\right\|^{2}=\delta_{k}^{2} .
$$

Lemma3.1 Remarking $g(u)=\left(1+\int_{\Omega}\left|D^{m} u\right|^{p} d x\right)^{r}(-\Delta)^{m} u, g: H_{0}^{2 m+k}(\Omega) \rightarrow H_{0}^{2 m}(\Omega)$ is uniformly bounded, and globally lipschitz continuous.

Proof. $\forall u_{1}, u_{2} \in H_{0}^{2 m+k}(\Omega)$

$$
\begin{aligned}
& g\left(u_{1}\right)-g\left(u_{2}\right)=\left(\left(1+\left\|D^{m} u_{1}\right\|_{p}^{p}\right)^{r}-\left(1+\left\|D^{m} u_{2}\right\|_{p}^{p}\right)^{r}\right) \cdot(-\Delta)^{m} u_{1}+\left(1+\left\|D^{m} u_{2}\right\|_{p}^{p}\right)^{r}(-\Delta)^{m}\left(u_{1}-u_{2}\right) . \\
& \qquad D^{k}\left(g\left(u_{1}-g\left(u_{2}\right)\right) \|\right. \leq g^{\prime}(\xi) \cdot\left\|D^{m+k} u_{1}-D^{m+k} u_{2}\right\| \cdot D^{2 m+k} u_{1}+g\left(u_{2}\right)\left\|D^{2 m+k}\left(u_{1}-u_{2}\right)\right\| \\
& \leq g^{\prime}(\xi) \cdot \lambda_{1}^{\frac{m}{2}}\left\|D^{2 m+k}\left(u_{1}-u_{2}\right)\right\|\left\|D^{2 m+k} u_{1}\right\|+g\left(u_{2}\right)\left\|D^{2 m+k}\left(u_{1}-u_{2}\right)\right\| \\
& \leq C_{0} \| D^{2 m+k}\left(u_{1}-u_{2}\right) .
\end{aligned}
$$

where $\xi=\theta D^{m} u_{1}+(1-\theta) D^{m} u_{2}, \theta \in[0,1]$. Let $l=C_{0}$, then $l$ is the lipschitz coefficient of $g(u)$.

Theorem 3.1 When $0<\beta \leq 2$, l is the lipschitz coefficient of $g\left(\left\|D^{m} u\right\|_{p}^{p}\right)$, let $N_{1} \in N$ make that $N \geq N_{1}$, we have

$$
\beta\left(\delta_{N+1}-\delta_{N}\right) \geq 8 l .
$$

Then the operator $\widetilde{A}$ satisfies the (7) spectral interval condition. 
Proof. According to equations (12) and (14), writing $U=(u, v), V=(\widehat{u}, \widehat{v}) \in X$, then

$$
\|F(U)-F(V)\|_{X}=\left\|g\left(\left\|D^{m} u\right\|_{p}^{p}\right)-g\left(\left\|D^{m} \widehat{u}\right\|_{p}^{p}\right)\right\| \leq l\|u-\widehat{u}\| \leq \frac{l}{\sqrt{\beta-2}}\|U-V\|_{X} .
$$

That is $l_{F} \leq l$. According to equation (19), the necessary and sufficient condition for $\lambda_{k}^{ \pm}$to be $\lambda_{k}^{ \pm}$real number that is $\beta>2$. By assuming that $0<\beta \leq 2, \widetilde{A}$ have at most $\lambda_{k}^{ \pm}$finite number of $2 N_{0}$ as eigenroots, when $N_{0}=0,0<\beta \leq 2$, then $\Lambda_{0}=\max \left\{\lambda_{k}^{ \pm} \mid k \leq N_{0}\right.$. When $k \geq N_{0}+1$, the eigenvalue is complex, and the real part is taken

$$
\operatorname{Re} \lambda_{k}^{ \pm}=\frac{\beta}{2} \delta_{k}
$$

So there is $N_{1} \geq N_{0}+1$ make $\operatorname{Re} \lambda_{k}^{ \pm}>\Lambda_{0}, k \geq N_{1}$.

Let make (22) be true. Decompose the dot spectrum of $\widetilde{A}$

$$
\sigma_{1}=\left\{\lambda_{k}^{ \pm} \mid k \leq N\right\}, \sigma_{2}=\left\{\lambda_{k}^{ \pm} \mid k \geq N+1\right\} .
$$

Let's set the corresponding subspace

$$
X_{1}=\operatorname{span}\left\{\lambda_{k}^{ \pm} \mid k \leq N\right\}, X_{2}=\operatorname{span}\left\{\lambda_{k}^{ \pm} \mid k \geq N+1\right\} .
$$

Inexistence $k$ make $\delta_{k}^{-} \in \sigma_{1}$ and $\delta_{k}^{+} \in \sigma_{2}$, which means it can't exist $U_{k}^{-} \in X_{1}$ and $U_{k}^{+} \in X_{2}$. Therefore, $X_{1}$ and $X_{2}$ are the normal subspace of $X$. According to (5) and (25), we get

$$
\operatorname{Re}\left(\lambda_{N+1}^{-}-\lambda_{N}^{+}\right)=\frac{\beta}{2}\left(\delta_{N+1}-\delta_{N}\right)
$$

Therefore, it can be known from (23) that $\widetilde{A}$ satisfies the spectral interval condition.

Theorem 3.2 When $\beta \geq 2$, l is the Lipschtiz coefficient of $g\left(\left\|D^{m} u\right\|_{p}^{p}\right)$ let $N_{1} \in N$ be sufficiently large, so that $N \geq N_{1}$ and

$$
\left(\delta_{N+1}-\delta_{N}\right)\left(\frac{\beta}{2}-\frac{\sqrt{\beta^{2}-4}}{2}\right) \geq \frac{4 l}{\sqrt{\beta-2}}+1
$$

Then operator $\widetilde{A}$ satisfies the spectral interval condition of (7).

Proof. When $\beta>2$, all eigenvalues of $\widetilde{A}$ are positive real numbers, and we know that the sequences $\left\{\lambda_{k}^{-}\right\}_{k \geq 1}$ and $\left\{\lambda_{k}^{+}\right\}_{k \geq 1}$ are increasing.The following are four steps to prove Theorem 3.2. Step 1: Because $\lambda_{k}^{ \pm}$is non-subtractive, according to Lemma 2.1, $N$ is given so that $\lambda_{N}^{-}$and $\lambda_{N+1}^{-}$are adjacent values, and the eigenvalue of $\tilde{A}$ is decomposed to

$$
\begin{array}{r}
\sigma_{1}=\left\{\lambda_{j}^{-}, \lambda_{k}^{+} \mid \max \left\{\lambda_{j}^{-}, \lambda_{k}^{+}\right\} \leq \lambda_{N}^{-}\right\}, \\
\sigma_{2}=\left\{\lambda_{j}^{-}, \lambda_{k}^{+} \mid \lambda_{j}^{-} \leq \lambda_{N}^{-} \leq \min \left\{\lambda_{j}^{-}, \lambda_{k}^{+}\right\}\right\} .
\end{array}
$$

Step 2: Corresponding $X$ can be decomposed into

$$
\begin{aligned}
& X_{1}=\operatorname{span}\left\{U_{j}^{-}, U_{k}^{+} \mid \lambda_{j}^{-}, \lambda_{k}^{+} \in \sigma_{1}\right\}, \\
& X_{2}=\operatorname{span}\left\{U_{j}^{-}, U_{k}^{+} \mid \lambda_{j}^{-}, \lambda_{k}^{+} \in \sigma_{2}\right\} .
\end{aligned}
$$

The goal is to make these two subspaces orthogonal and satisfy the interspectral expression (7). $\Lambda_{1}=\lambda_{N}^{-}, \Lambda_{2}=\lambda_{N+1}^{-}$, Further decomposition $X_{2}=X_{C} \oplus X_{R}$,

$$
\begin{array}{r}
X_{C}=\operatorname{span}\left\{U_{j}^{-} \mid \lambda_{j}^{-} \leq \lambda_{N}^{-} \leq \lambda_{j}^{+}\right\}, \\
X_{R}=\operatorname{span}\left\{U_{R}^{ \pm} \mid \lambda_{N}^{-} \leq \lambda_{k}^{ \pm}\right\} .
\end{array}
$$

And assuming that $X_{N}=X_{C} \oplus X_{1}$. Next, specify the dot product of the eigenvalues over $X$, so that $X_{1}$ and $X_{2}$ are orthogonal, so we need to introduce two functions $\Phi: X_{N} \rightarrow R$ and $\Psi: X_{R} \rightarrow R$.

$$
\begin{array}{r}
\Phi(U, V)=(\beta-1)\left((-\Delta)^{m} u,(-\Delta)^{m} \bar{y}\right)+\left(\bar{z},(-\Delta)^{m} u\right)+\left(\bar{v},(-\Delta)^{m} y\right)+(\bar{z}, v), \\
\Psi(U, V)=\beta\left((-\Delta)^{m} u,(-\Delta)^{m} \bar{y}\right)+\left(\bar{z},(-\Delta)^{m} u\right)+\left(\bar{v},(-\Delta)^{m} y\right)+(\bar{z}, v) .
\end{array}
$$


Where $U=(u, v), V=(y, z), \bar{y}$ and $\bar{z}$ are conjugates of $y$ and $z$, respectively.

Suppose $U=(u, v) \in X_{N}$, then

$$
\begin{aligned}
\Phi(U, U) & =(\beta-1)\left\|(-\Delta)^{m} u\right\|^{2}+2\left(\bar{v},(-\Delta)^{m} u\right)+\|v\|^{2} \\
& \geq(\beta-1)\left\|(-\Delta)^{m} u\right\|^{2}-2\|v\| \cdot\left\|(-\Delta)^{m} u\right\|+\|v\|^{2} \\
& \geq(\beta-1)\left\|(-\Delta)^{m} u\right\|^{2}-\|v\|^{2}-\left\|(-\Delta)^{m} u\right\|^{2}+\|v\|^{2} \\
& \geq(\beta-2)\left\|(-\Delta)^{m} u\right\|^{2} .
\end{aligned}
$$

And since $\beta>2$, you get that $\Phi(U, U) \geq 0$ is true for all $U=(u, v) \in X_{N}, \Phi$ is positive definite.

Similarly, since $U=(u, v) \in X_{R}$,

$$
\begin{aligned}
\Psi(U, U) & =\beta\left\|(-\Delta)^{m} u\right\|^{2}+2\left(\bar{v},(-\Delta)^{m} u\right)+\|v\|^{2} \\
& \geq \beta\left\|(-\Delta)^{m} u\right\|^{2}-2\|v\| \cdot\left\|(-\Delta)^{m} u\right\|+\|v\|^{2} \\
& \geq \beta\left\|(-\Delta)^{m} u\right\|^{2}-\|v\|^{2}-\left\|(-\Delta)^{m} u\right\|^{2}+\|v\|^{2} \\
& \geq(\beta-1)\left\|(-\Delta)^{m} u\right\|^{2} .
\end{aligned}
$$

And since $\beta>2$, you get that $\Psi(U, U) \geq 0$ is true for all $U=(u, v) \in X_{N}, \Psi$ is also positive definite.

Now we're going to specify the inner product of $X$

$$
\ll U, V \gg_{X}=\Phi\left(P_{N} U, P_{N} V\right)+\Psi\left(P_{R} U, P_{R} V\right) .
$$

Where $P_{N}$ and $P_{R}$ are respectively mappings of $X \rightarrow X_{N}$ and $X \rightarrow X_{R}$. For convenience, equation (40) can be rewritten to obtain

$$
\ll U, V \gg_{X}=\Phi(U, V)+\Psi(U, V) .
$$

It will be shown that the two subspaces $X_{1}$ and $X_{2}$ defined in (32) and (33) are orthogonal with respect to the dot product (41).In fact, $X_{N}$ and $X_{C}$ are orthogonal, that is $\ll U_{j}^{+}, U_{j}^{-} \gg_{X}=0$, For each $U_{j}^{+} \in X_{C}$ and $U_{j}^{-} \in x_{N}$, we can deduce from Equation (35)

$$
\begin{aligned}
\ll U_{j}^{+}, U_{j}^{-} \gg_{X} & =\Phi\left(U_{j}^{+}, U_{j}^{-}\right) \\
& =(\beta-1)\left\|(-\Delta)^{m} u_{j}\right\|^{2}-\left(\lambda_{j}^{-}+\lambda_{j}^{+}\right)\left\|D^{m} u_{j}\right\|^{2}+\lambda_{j}^{-} \lambda_{j}^{+}\left\|u_{j}\right\|^{2} \\
& =(\beta-1) \delta_{j}^{2}-\left(\lambda_{j}^{-}+\lambda_{j}^{+}\right) \delta_{j}+\lambda_{j}^{-} \lambda_{j}^{+} .
\end{aligned}
$$

According to equation (18),we get $\lambda_{j}^{-}+\lambda_{j}^{+}=\beta \delta_{j}, \lambda_{j}^{-} \lambda_{j}^{+}=\delta_{j}^{2}$, so

$$
\ll U_{j}^{+}, U_{j}^{-} \gg_{X}=\Phi\left(U_{j}^{+}, U_{j}^{-}\right)=0 .
$$

Step 3: Next estimate the lipschitz constant of $F$, where $F(U)=(0, f(x)-g(u))^{T}, g: H^{2 m+k} \rightarrow H^{2 m}$ and $l_{F}=l$. From equation (30) and equation (31), it can be seen that for any $U=(u, v) \in X$, we have

$$
\begin{aligned}
\|U\|_{X}^{2} & =\Phi\left(P_{1} U, P_{1} U\right)+\Psi\left(P_{2} U, P_{2} U\right) \\
& \geq(\beta-2)\left(\left\|(-\Delta)^{m} P_{1} u\right\|^{2}+\left\|(-\Delta)^{m} P_{2} u\right\|^{2}\right) \\
& \geq(\beta-2)\left\|(-\Delta)^{m} u\right\|^{2} .
\end{aligned}
$$

Set $U=(u, v), V=(\bar{u}, \bar{v}) \in X$, we have

$$
\|F(U)-F(V)\|_{X}=\left\|g\left(\left\|D^{m} u\right\|_{p}^{p}\right)-g\left(\left\|D^{m} \bar{u}\right\|_{p}^{p}\right)\right\| \leq l\|u-\bar{u}\| \leq \frac{l}{\sqrt{\beta-2}}\|U-V\|_{X} .
$$

So we get the conclusion

$$
l_{F} \leq \frac{l}{\sqrt{\beta-2}}
$$


Step 4: The spectral interval condition formula (7) needs to be verified, which can be obtained from $\Lambda_{1}=\lambda_{N}^{-}$and $\Lambda_{2}=\lambda_{N+1}^{-}$mentioned above

$$
\Lambda_{2}-\Lambda_{1}=\lambda_{N+1}^{-}-\lambda_{N}^{-}=\frac{1}{2}\left(\delta_{N+1}-\delta_{N}\right)+\frac{1}{2}(\sqrt{R(N)}-\sqrt{R(N+1)}) .
$$

Where $R(N)=\beta^{2} \delta_{N}^{2}-4 \delta_{N}^{2}$.

Make sure that $N_{1}>0$ is such that for all $N \geq N_{1}, R_{1}(N)=1+\frac{2 \beta}{\left(\beta^{2}-4\right) \delta_{N}^{2}}+\frac{1}{\left(\beta^{2}-4\right) \delta_{N}^{2}}$, so we can calculate

$$
\sqrt{R(N)}-\sqrt{R(N+1)}+\sqrt{\beta-2}\left(\delta_{N+1}-\delta_{N}\right)=\sqrt{\beta-2}\left(\delta_{N+1} R_{1}(N+1)-\delta_{N} R_{1}(N)\right) .
$$

Well, it's easy to know from the assumptions that we've made

$$
\lim _{N \rightarrow \infty}\left(\sqrt{R(N)}-\sqrt{R(N+1)}+\sqrt{\beta-2}\left(\delta_{N+1}-\delta_{N}\right)\right)=0 .
$$

Then, by combining equations (46), (47), (21) and (48), we can obtain

$$
\Lambda_{2}-\Lambda_{1}>\frac{1}{2}\left(\left(\delta_{N+1}-\delta_{N}\right)\left(\beta-\sqrt{\beta^{2}-4}\right)-1\right) \geq \frac{4 l}{\sqrt{\beta-2}} \geq 4 l_{F} .
$$

\section{References}

Chueshov, I. D. (1996). On a construction of approximate inertial manifolds for second order in time evolution equations. Nonlinear Analysis: Theory, Methods E Applications, 26(5), 1007-1021.

Ciprian, F. (1998). Inertial manifolds for nonlinear evalutionary equations. Journal of Differential Equations, 309-353.

Guoguang, L. (2011). Nonlinear evolution equation. Yunnan university.

Guoguang, L. (2019). Kinetic properties of serval Kirchhoff equations. Chongqing university (pp: 139-181).

Jolly, M. S., Kevrekidis, I. G., \& Titi, E. S. (1990). Approximate inertial manifolds for the Kuramoto-Sivashinsky equation: analysis and computations. Physica D: Nonlinear Phenomena, 44(1-2), 38-60.

Margolin, L. G., \& Jones, D. A. (1992). An approximate inertial manifold for computing Burgers' equation. Physica D: Nonlinear Phenomena, 60(1-4), 175-184.

Marion, M. (1989). Inertial manifolds associated to partly dissipative reaction-diffusion systems. Journal of mathematical analysis and applications, 143(2), 295-326.

Temam, R. (1990). Inertial manifolds. The Mathematical Intelligencer, 12(4), 68-74.

Wu, J. Z., \& Lin, G. G. (2010). An inertial manifold of the two-dimensional strongly damped Boussinesq equation. Journal of Yunnan University (Natural Science Edition), 32, 119-224.

Wu, J. Z., Zhao, P., \& Lin, G. G. (2010). An inertial manifold of the damped Boussinesq equation. Journal of Yunnan University (Natural Sciences Edition), S1.

Youwei, L., Bixiang, W., \& Bingshen, Y. (1997). Regularity and Approximate Inertial Manifolds for a Class of Evalutionary Equations. Journal of Lanzhou Univerity:Natural Science, 33(1), 10-16.

Zhicheng, Z., \& Guoguang, L. (2013). An inertial manifold of strongly damped delay wave equation of the fourth order.Journal of Yunnan university, 35(S1), 164-171.

\section{Copyrights}

Copyright for this article is retained by the author(s), with first publication rights granted to the journal.

This is an open-access article distributed under the terms and conditions of the Creative Commons Attribution license (http://creativecommons.org/licenses/by/4.0/). 